\title{
Unintegrated CCFM parton distributions and transverse momentum of gauge bosons
}

\author{
J. Kwieciński $^{1}$ and A. Szczurek ${ }^{1,2}$ \\ ${ }^{1}$ Institute of Nuclear Physics \\ PL-31-342 Cracow, Poland \\ 2 University of Rzeszów \\ PL-35-959 Rzeszów, Poland
}

\begin{abstract}
Transverse momentum distribution of gauge bosons $W^{ \pm}$and $Z^{0}$ is calculated using unintegrated parton distributions obtained by solving leading order CCFM equation(s) in the impact parameter space. For illustration we compare the results of the fixed-order collinear, softgluon resummation and CCFM approaches. A parameter of a nonperturbative form factor is adjusted to the D0 data. In contrast to the collinear approach, the leading order calculation based on the unintegrated CCFM parton distributions generates transverse momentum distributions of gauge bosons, which are almost consistent with experimental data provided the parameter of the nonperturbative form factor is appropriately adjusted. The $W^{+}-W^{-}$asymmetry is calculated as a function of the boson rapidity and transverse momentum. Predictions are given for RHIC and LHC.
\end{abstract}

\section{Introduction}

The production of gauge bosons at hadron colliders provides fundamental tests of perturbative QCD. The understanding of transverse momentum distribution is of particular interest in this context. The data from the Tevatron collider cover regions of both small and large gauge-boson transverse momenta $\left(p_{t}\right)$ [1, 2, 3]. At large $p_{t}$ the standard fixed-order pQCD approach is adequate. At small $p_{t}$ of vector bosons $\left(p_{t} \ll M_{V}\right)$ one encounters large logarithms of $M_{V}^{2} / p_{t}^{2}$. The leading term is of the form

$$
\frac{d \sigma}{d p_{t}^{2}} \sim \frac{\alpha_{s}}{p_{t}^{2}} \ln \frac{M_{V}^{2}}{p_{t}^{2}}
$$


The logarithms can be resummed to give a Sudakov form factor. In recent years the resummation approach became the standard in calculating transverse momentum distribution of gauge-bosons [4, 5, 6, 7, 8, 9. The most often used is the formalism proposed by Collins, Soper and Sterman 4. In the following we shall refer to it as CSS or "the b-space resummation approach". The b-space resummation approach is subjected to an ambiguity in setting the matching condition between the resummed and fixed-order results. Different prescriptions have been used throughout the literature.

The collinear parton approach was used by a few specialized group to calculated many high-energy observables. Not all of them can be fully explained in the fixed-order collinear parton model. The $k_{t}$-factorization approach, using unintegrated parton distributions, is an alternative approach. In recent years there were many calculations of specific observables using different sets of unintegrated parton distributions. Mainly observables sensitive to unintegrated gluon distributions have been discussed, like the dijet or heavy-quark pair production in high-energy electron-proton scattering at HERA or the production of heavy quark pairs in hadron-hadron collisions at high energy. The existing unintegrated gluon distribution differ one from another. In some approaches (models) the unintegrated gluon distributions are limited to the small- $x$ region by construction. The unintegrated (anti)quark distributions are very sparse and often not available. Clearly, the program of a systematic study of the world data in terms of unintegrated parton distributions (uPDFs) is at its beginning.

Gluon radiation along the ladder generates transverse momentum of partons through the recoil effect. Evolution of the unintegrated distributions is described then by the so-called CCFM equation [10. The CCFM equation has a nice virtue that it interpolates between the BFKL evolution at very small values of $x$ and the DGLAP evolution at intermediate and large values of $x$. For many practical applications it is very useful to solve the CCFM equation by means of a Monte Carlo method [15. The CCFM approach turns out very succesful in resolving some high-energy puzzles [16, 17, 18, 19. In the so-called b-representation the CCFM equation for b-dependent unintegrated distributions takes a simple diagonal form [11. This approach can be generalized to include in addition to unintegrated gluon distributions also unintegrated (anti)quark distributions [13].

The similarities and differences betwen the standard soft gluon resummation and the CCFM resummation have been discussed recently on the example of the $g g \rightarrow H$ fusion mechanism [14. It has been shown how the soft gluon resummation formulae can be obtained as the result of an approximate treatment of the solution of the CCFM equation in the so-called 
b-representation.

We shall show that in contrast to the collinear approach the use of uPDFs leads to a non- $\delta$-like transverse momentum distribution of the produced gauge bosons already in the leading order for matrix elements. It is our aim here to calculate the transverse momentum distribution of the charged gauge bosons based on the unintegrated parton distribution originating from the numerical solution of the CCFM equation. This will be a first phenomenological test of the CCFM parton distributions from [13. In addition, we wish to concentrate on the $W^{+}-W^{-}$asymmetry, especially for proton-proton collisions at RHIC and LHC. We shall show that this asymmetry strongly depends on the $\bar{d}-\bar{u}$ asymmetry of parton distributions, providing a new interesting tool to limit the latter somewhat better.

\section{Formalism}

In the fixed-order perturbative treatment the situation is as follows. In the 0th order collinear calculation the produced gauge bosons carry no transverse momentum, i.e.

$$
\begin{aligned}
& \frac{d \sigma}{d y d^{2} p_{t}}=\sigma_{0}^{W} \sum_{q q^{\prime}}\left|V_{q q^{\prime}}\right|^{2} \\
& {\left[x_{1} q_{1}\left(x_{1}, \mu^{2}\right) x_{2} \bar{q}_{2}^{\prime}\left(x_{2}, \mu^{2}\right)+x_{1} \bar{q}_{1}^{\prime}\left(x_{1}, \mu^{2}\right) x_{2} q_{2}\left(x_{2}, \mu^{2}\right)\right] \cdot \delta^{2}\left(\vec{p}_{t}\right) .}
\end{aligned}
$$

For brevity, above we have introduced $\sigma_{0}^{W}=\frac{2 \pi G_{F}}{3 \sqrt{2}}$. The quark/antiquark momentum distributions are evaluated at momentum fractions $x_{1,2}=\frac{M_{W}}{\sqrt{s}} \exp ( \pm y)$.

A nonzero transverse momentum of gauge bosons is obtained only in the next-to-leading order calculation. Then the gauge bosons with nonzero transverse momenta are produced in the weak-QCD annihilation or Compton processes in association with a gluon (annihilation) or quark/antiquark (Compton). The cross section associated with the $2 \rightarrow 2$ subprocess $(1+2 \rightarrow$ $\mathrm{W}+4)$ is given by the standard parton formula

$$
\frac{d \sigma}{d y_{3} d y_{4} d^{2} p_{t}}=\frac{1}{16 \pi^{2} \hat{s}^{2}} \sum_{i j} x_{1} p_{1}\left(x_{1}, \mu^{2}\right) x_{2} p_{2}\left(x_{2}, \mu^{2}\right) \bar{\sum}\left|M_{i j}\right|^{2},
$$

where the matrix elements squared, averaged over spins and colors of incoming partons and summed over spins and colors of final particles read:

$$
\sum_{q q^{\prime}}\left|M_{q \bar{q}^{\prime} \rightarrow W g}\right|^{2}=\pi \alpha_{s}\left(\mu_{r}^{2}\right) \sqrt{2} G_{F} M_{W}^{2}\left|V_{q q^{\prime}}\right|^{2} \frac{8}{9} \frac{\hat{t}^{2}+\hat{u}^{2}+2 M_{W}^{2} \hat{s}}{\hat{t} \hat{u}}
$$


for the annihilation and

$$
\sum_{q q^{\prime}}\left|M_{g q \rightarrow W q^{\prime}}\right|^{2}=\pi \alpha_{s}\left(\mu_{r}^{2}\right) \sqrt{2} G_{F} M_{W}^{2}\left|V_{q q^{\prime}}\right|^{2} \frac{1}{3} \frac{\hat{s}^{2}+\hat{u}^{2}+2 M_{W}^{2} \hat{t}}{-\hat{s} \hat{u}}
$$

for the weak Compton subprocess. In the equations above $V_{q q^{\prime}}$ are elements of the Cabibbo-Kobayashi-Maskawa matrix. The parton distributions are evaluated at $x_{1,2}=\frac{1}{\sqrt{s}} \cdot\left(m_{t, W} \exp \left( \pm y_{1}\right)+p_{t} \exp \left( \pm y_{2}\right)\right)$ where the transverse mass $m_{t, W}=\sqrt{M_{W}^{2}+p_{t}^{2}}$.

The poles at $\hat{t}=0$ or $\hat{u}=0$ cause the theoretical cross section to diverge as $p_{t} \rightarrow 0$ and exceeds the experimental data at $p_{t}<5 \mathrm{GeV}$ (see Fig (1)). Virtual corrections to $q \bar{q}^{\prime} \rightarrow W$ contribute only at $p_{t}=0\left(d \sigma^{W} / d p_{t}^{2} \propto \delta\left(p_{t}^{2}\right)\right)$, ensuring that the transverse momentum integrated cross section is finite, while leaving problems at small finite transverse momenta.

In the CCS approach [4] the resummed cross section for $W$ production can be written as

$$
\begin{array}{r}
\frac{d \sigma}{d y d^{2} p_{t, W}}=\sigma_{0}^{W} /(2 \pi)^{2} \sum_{q q^{\prime}}\left|V_{q q^{\prime}}\right|^{2} \int d^{2} b J_{0}\left(p_{t} b\right) W_{q \bar{q}^{\prime}}^{N P}\left(Q, b, x_{1}, x_{2}\right) \\
x_{1} \cdot\left[q_{1}\left(x_{1}, \mu(b)\right)+\frac{\alpha_{s}(\mu(b))}{2 \pi} C_{v c} q_{1}\left(x_{1}, \mu(b)\right)+\frac{\alpha_{s}(\mu(b))}{2 \pi}\left(C_{q g} \otimes g_{1}\right)\left(x_{1}, \mu(b)\right)\right] \\
x_{2} \cdot\left[\bar{q}_{2}^{\prime}\left(x_{2}, \mu(b)\right)+\frac{\alpha_{s}(\mu(b))}{2 \pi} C_{v c} \bar{q}_{2}^{\prime}\left(x_{2}, \mu(b)\right)+\frac{\alpha_{s}(\mu(b))}{2 \pi}\left(C_{\bar{q}^{\prime} g} \otimes g_{2}\right)\left(x_{2}, \mu(b)\right)\right] \\
\exp \left[\frac{1}{2}\left(S_{q}(b, Q)+S_{\bar{q}^{\prime}}(b, Q)\right)\right],
\end{array}
$$

where the exponents in the Sudakov-like form factors read

$$
S_{q}(b, Q)=S_{\bar{q}}(b, Q)=-\int_{\bar{\mu}_{\min }^{2}}^{Q^{2}} \frac{d \bar{\mu}^{2}}{\bar{\mu}^{2}}\left[\ln \left(\frac{Q^{2}}{\bar{\mu}^{2}}\right) A_{q}\left(\alpha_{s}(\bar{\mu})\right)+B_{q}\left(\alpha_{s}(\bar{\mu})\right)\right] .
$$

The coefficient $A$ and $B$ can be expanded in the series of $\alpha_{s}$ :

$$
\begin{aligned}
A_{q} & =2 C_{F}+\frac{\alpha_{s}(\bar{\mu})}{2 \pi}(\ldots)+\ldots, \\
B_{q} & =-3 C_{F}+\frac{\alpha_{s}(\bar{\mu})}{2 \pi}(\ldots)+\ldots .
\end{aligned}
$$

The CSS formalism [4] leaves open the question of small $b$. Different prescriptions have been proposed to treat this region (see e.g. 7]). The lower 
limit of the integral in Eq.(17) is usually taken $\mu_{\min }^{2}=\left(\frac{C_{b}}{b}\right)^{2}$, where $C_{b}=2$ $\exp \left(-\gamma_{E}\right) \approx 1.1229$. This prescription leads to a kink for the Sudakov form factor if $C_{b} / b=Q$. In order to allow a smooth dependence of the cross section on the gauge-boson transverse momentum and to quarantee that the lower limit is really lower than the upper limit, one could make the following replacement $\mu_{\text {min }}^{2}=\left(\frac{C_{b}}{b}\right)^{2} \rightarrow\left(\frac{C_{b}}{b}\right)^{2}\left[1+C_{b}^{2} /\left(b^{2} Q^{2}\right)\right]^{-1}$ (see e.g. [7]). In order to quarantee that the scale of parton distribution does not take unphysically small values we shall use in addition the following prescription:

$$
\mu^{2}(b)=\mu_{m i n}^{2}+\mu_{0}^{2}
$$

where $\mu_{0}^{2} \gg \Lambda_{Q C D}^{2}$ is the starting value for the QCD evolution. For illustration, in the present paper we shall use easy to handle the leading order parton distributions from Ref.[20].

In the formalism of unintegrated parton distributions the nonzero transverse momenta of gauge bosons are obtained already in the leading order. The invariant cross section for inclusive gauge boson production reads then as

$$
\begin{gathered}
\frac{d \sigma}{d y d^{2} p_{t, W}}=\sigma_{0}^{W} \sum_{q q^{\prime}}\left|V_{q q^{\prime}}\right|^{2} \int \frac{d^{2} \kappa_{1}}{\pi} \frac{d^{2} \kappa_{2}}{\pi} \delta^{2}\left(\vec{p}_{t}-\vec{\kappa}_{1}-\vec{\kappa}_{2}\right) \\
{\left[f_{q / 1}\left(x_{1}, \kappa_{1}^{2}, \mu^{2}\right) f_{\bar{q}^{\prime} / 2}\left(x_{2}, \kappa_{2}^{2}, \mu^{2}\right)+f_{\bar{q}^{\prime} / 1}\left(x_{1}, \kappa_{1}^{2}, \mu^{2}\right) f_{q / 2}\left(x_{2}, \kappa_{2}^{2}, \mu^{2}\right)\right] .}
\end{gathered}
$$

In the equation above the delta function assures the conservation of transverse momenta in the $q \bar{q}^{\prime}$ fusion subprocess. The momentum fractions are calculated as $x_{1,2}=\frac{m_{t, W}}{\sqrt{s}} \exp ( \pm y)$, where in contrast to the collinear case $M_{W}$ is replaced by the transverse mass $m_{t, W}$. In the case of the gauge boson production, the scale $\mu^{2}$ is taken as $M_{W}^{2}$ or $M_{Z}^{2}$.

We note that formally, as far as matrix elements are considered, formula (10) is the $k_{t}$-factorization counterpart of the collinear formula (2).

Introducing unintegrated parton distributions in the space conjugated to the transverse momenta [1] ${ }^{1}$

$$
f_{q}\left(x, \kappa^{2}, \mu^{2}\right)=\frac{1}{2 \pi} \int \exp (i \vec{\kappa} \vec{b}) \tilde{f}_{q}\left(x, b, \mu^{2}\right) d^{2} b,
$$

and taking the following representation of the $\delta$ function

$$
\delta^{2}\left(\overrightarrow{\kappa_{1}}+\overrightarrow{\kappa_{2}}-\overrightarrow{p_{t}}\right)=\frac{1}{(2 \pi)^{2}} \int d^{2} b \exp \left[\left(\overrightarrow{\kappa_{1}}+\overrightarrow{\kappa_{2}}-\overrightarrow{p_{t}}\right) \vec{b}\right],
$$

\footnotetext{
${ }^{1}$ In the present paper we shall use the notation $\tilde{f}$ instead of $\bar{f}$ as in Refs. [13, 14] to avoid confusion with antiquark distributions needed here.
} 
the formula (10) can be written in the equivalent way

$$
\begin{gathered}
\frac{d \sigma}{d y d^{2} p_{t, W}}=\sigma_{0}^{W} / \pi^{2} \sum_{q q^{\prime}}\left|V_{q q^{\prime}}\right|^{2} \int d^{2} b J_{0}\left(p_{t} b\right) \\
{\left[\tilde{f}_{q / 1}\left(x_{1}, b, \mu^{2}\right) \tilde{f}_{\bar{q}^{\prime} / 2}\left(x_{2}, b, \mu^{2}\right)+\tilde{f}_{\bar{q}^{\prime} / 1}\left(x_{1}, b, \mu^{2}\right) \tilde{f}_{q / 2}\left(x_{2}, b, \mu^{2}\right)\right] .}
\end{gathered}
$$

In the next section we show and discuss the results obtained with the formula (13). In the formulae for $Z^{0}$ boson production $\left|V_{q q^{\prime}}\right|^{2}$ is replaced by $\delta_{q q^{\prime}} \frac{1}{2}\left(V_{q}^{2}+\right.$ $\left.A_{q}^{2}\right)$.

As already mentioned in the introduction, it is our intention here to use uPDFs $\tilde{f}_{q}^{C C F M}\left(x, b, \mu^{2}\right)$ which fulfill b-space CCFM equations [1], 12]. However, the perturbative solutions $\tilde{f}_{q}^{C C F M}\left(x, b, \mu^{2}\right)$ do not include nonperturbative effects such as, for instance, intrinsic momentum distribution of partons in colliding hadrons. In order to include such effects we propose to modify the perturbative solution $\tilde{f}_{q}^{C C F M}\left(x, b, \mu^{2}\right)$ and write the modified parton distributions $\tilde{f}_{q}\left(x, b, \mu^{2}\right)$ in the simple factorized form

$$
\tilde{f}_{q}\left(x, b, \mu^{2}\right)=\tilde{f}_{q}^{C C F M}\left(x, b, \mu^{2}\right) \cdot F_{q}^{N P}(b) .
$$

In the present study we shall use two different functional forms for the form factor

$$
F_{q}^{N P}(b)=F^{N P}(b)=\exp \left(-\frac{b^{2}}{4 b_{0}^{2}}\right) \text { or } \exp \left(-\frac{b}{b_{e}}\right)
$$

identical for all species of partons. In Eq.(15) $b_{0}$ (or $\left.b_{e}\right)$ is the only free parameter. In the next section we try to adjust this parameter to the experimental data on transverse momentum distribution of $W^{ \pm}$.

We wish to note an almost identical structure of the b-space resummation formula (6) and our formula (13). Assuming a factorizable form for the nonperturbative form factor $W_{q \bar{q}^{\prime}}^{N P}$ :

$$
W_{q \bar{q}^{\prime}}^{N P}\left(Q, b, x_{1}, x_{2}\right)=F_{q}^{N P}\left(Q, b, x_{1}\right) \cdot F_{\bar{q}^{\prime}}^{N P}\left(Q, b, x_{2}\right),
$$

the formulae (6) and (13) are identical as far as the formal structure is considered, if we define effective b-space unintegrated (anti)quark distributions

$$
\begin{aligned}
& \tilde{f}_{q_{1}}^{S G R}\left(x_{1}, b, Q^{2}\right)=\frac{1}{2} F_{q}^{N P}\left(Q, b, x_{1}\right)\left[x_{1} q_{1}\left(x_{1}, \mu(b)\right)+\ldots\right] \exp \left(\frac{1}{2} S_{q}(b, Q)\right) \\
& \tilde{f}_{\bar{q}_{2}^{\prime}}^{S G R}\left(x_{2}, b, Q^{2}\right)=\frac{1}{2} F_{\bar{q}^{\prime}}^{N P}\left(Q, b, x_{2}\right)\left[x_{2} \bar{q}_{1}^{\prime}\left(x_{2}, \mu(b)\right)+\ldots\right] \exp \left(\frac{1}{2} S_{\bar{q}^{\prime}}(b, Q)() 7 .\right)
\end{aligned}
$$

The index $S G R$ above stands for "soft-gluon resummation". The $k_{t}$-dependent unintegrated distributions of (anti)quarks corresponding to the b-space resummation can be then obtained through the Fourier-Bessel transform

$$
f_{q}^{S G R}\left(x, \kappa^{2}, Q^{2}\right)=\int d b b J_{0}(\kappa b) \tilde{f}_{q}^{S G R}\left(x, b, Q^{2}\right) .
$$


With the simple Ansatz (15) for $F_{q}^{N P}$ the whole $Q^{2}$ dependence resides exclusively in the Sudakov-like form factor.

\section{Results}

For further reference in Fig 1 we present results of the collinear NLO calculation. In this calculation the argument of the running strong coupling constant $\mu_{r}^{2}=M_{W}^{2}$ and the scale argument is $\mu^{2}=M_{W}^{2}$. In panel (a) we show the low- $p_{t}$ region, whereas in panel (b) the whole measured region. The low- $p_{t}$ problems are clearly visible. At the Fermilab energy the annihilation contribution dominates over the Compton one in the entire measured region. The $2 \rightarrow 2$ NLO contribution underestimates the experimental data, especially in the interval $5 \mathrm{GeV}<p_{t}<50 \mathrm{GeV}$.

In our CCFM calculation we have taken $\mu^{2}=M_{W}^{2}$ as the factorization scale in the b-space unintegrated parton distributions. In Fig 2 we present a two-dimensional map $\left(y, p_{t}\right)$ of the invariant cross section. Large transverse momenta of $\mathrm{W}$ bosons are generated in the leading order calculations, which is due to transverse momenta of fusing partons contained in unintegrated (anti)quark distributions.

In Fig 3 and Fig 4 we show transverse momentum distribution (integrated over rapidities) of $W^{ \pm}$in proton-antiproton collissions at Fermilab at $\mathrm{W}=1.8$ $\mathrm{TeV}$ for the CCFM parton distributions (left panel) and standard soft-gluon resummation (right panel). The three curves in Fig 3 show results obtained according to formula (13) with different values of the Gaussian form factor parameter $b_{0}: b_{0}=0.5 \mathrm{GeV}^{-1}$ (dashed), $b_{0}=1.0 \mathrm{GeV}^{-1}$ (solid) and $b_{0}=2.0$ $\mathrm{GeV}^{-1}$. Similarly, the curves in Fig 4 correspond to $b_{e}=0.5 \mathrm{GeV}^{-1}$ (dashed), $b_{e}=1.0 \mathrm{GeV}^{-1}$ (solid) and $b_{e}=2.0 \mathrm{GeV}^{-1}$ in the exponential form factor. The results are overimposed on the D0 collaboration data [3] measured at Fermilab. In order to convert the measured cross section ( $e \nu$ channel) to the cross section for $\mathrm{W}$ production we have taken the corresponding branching ratio $B R(e \nu)=0.1073$ [3. For the Gaussian form factor (see Fig [3) somewhat better agreement with the data is obtained with the CCS approach, while for the exponential form factor (see Fig 4) it is the opposite. One should remember, however, that the situation may change if $2 \rightarrow 2$ NLO processes, neglected here, are included to the CCFM formalism. In the region of large momenta those contributions are expected to be similar in size to the collinear NLO contributions shown in Fig 1 (this is roughly the missing strength at $p_{t}$ $=20 \mathrm{GeV}$ ). In the CCS approach one somewhat arbitrarily matches the softgluon resummation result with the fixed order collinear result at the certain (chosen ad hoc) value of $p_{t}$. We hope that in the formalism of unintegrated 
parton distributions in the next-to-leading order approximation this can be done less arbitrarily. The $D 0$ data for $\mathrm{W}$ production are not precise enough to determine the parameter $b_{0}$ (or $b_{e}$ ) of the nonperturbative form factor with high precision. The low- $p_{t} D 0$ data for production of $\mathrm{Z}$ boson should be better in this respect.

In Fig 5 we show transverse momentum distribution (rapidity integrated) of $Z$ bosons in proton-antiproton collisions at Fermilab at $\mathrm{W}=1.8 \mathrm{TeV}$. As for the $\mathrm{W}$ production the theoretical results are compared with the D0 experimental data 1. We have chosen the somewhat older data set [1] instead of the most recent one [3] because of the binning with slightly better resolution at small $p_{t}$. In order to convert the measured cross section ( $e^{+} e^{-}$channel) to the cross section for $\mathrm{Z}$ production we have taken the corresponding branching ratio $B R\left(e^{+} e^{-}\right)=0.033632$ 3. The $\mathrm{D} 0$ data allow better extraction of the parameter $b_{0}$ of the nonperturbative form factor $F_{n p}$. By comparison to the data we find $b_{0}=0.5-1.0 \mathrm{GeV}^{-1}$ (Gaussian) as giving the best description of the low- $p_{t} Z^{0}$-production data.

Comparing Fig [1] and Figs 3 and 4 one observes that the leading-order CCFM approach generates more W's with $10 \mathrm{GeV}<p_{t}<20 \mathrm{GeV}$ than the standard next-to-leading order collinear approach. The leading-order CCFM approach leaves some room at $p_{t}>5 \mathrm{GeV}$. This is especially visible for the Gaussian form factor. In this context it would be very interesting to calculate the cross sections with the help of CCFM uPDF's up to the next-to-leading order.

In the future, the gauge bosons will be measured also in proton-proton collisions at the BNL RHIC and at the CERN LHC. As an example of our predictions in Fig [ we present the transverse momentum distribution of $W^{ \pm}$ for three different center-of-mass energies $\mathrm{W}=200,500,14000 \mathrm{GeV}$. In this calculation $-2<y<2$ and the parameter $b_{0}$ was fixed at $1 \mathrm{GeV}^{-1}$. The larger energy, the smaller- $x$ region of (anti)quark distributions is probed. In principle, the form factor in (15) can be not only a function of impact parameter $b$, but also of partonic momentum fraction $x$. Having precise data at different energies could help then to pin down the $x$ dependence of the nonperturbative form factor. Other partonic processes could also be helpful in this respect.

The precise determination of parton distributions is one of the most important goals of high-energy physics. It was suggested in Ref. 22. that studies of the difference in the production of $W^{+}$and $W^{-}$in proton-proton collisions could put further constraints on the $\bar{d}-\bar{u}$ asymmetry as obtained from other sources, like deep-inelastic inclusive and exclusive scattering or Drell-Yan processes. In this illustrative leading order calculations, however, one could not address the problem of transverse momentum dependence of the $W^{-} / W^{+}$ 
ratio. In contrast, it is very simple in our leading-order CCFM approach. In Fig [7] we present the ratio as a function of W-boson transverse momentum at a typical RHIC energy $W=500 \mathrm{GeV}$ for selected $\mathrm{W}$-boson rapidities $\mathrm{y}=0$ (left panel) and $\mathrm{y}= \pm 1$ (right panel). The solid curve corresponds to the calculation with the $\bar{d}-\bar{u}$ asymmetry, while the dashed curve shows the result with $\bar{d}-\bar{u}$ asymmetry switched off. In general, the ratio $R=\sigma\left(W^{-}\right) / \sigma\left(W^{+}\right)$ is smaller if the $\bar{d}-\bar{u}$ asymmetry is included. The relative effect is larger for $\mathrm{y}=0$. Fig. [7 illustrates therefore the potential to study the $\bar{d}-\bar{u}$ asymmetry in $\mathrm{W}$-boson production at RHIC. There is only a very weak dependence on W-boson transverse momentum. In order to illustrate the dependence on W-boson rapidity in Fig 8 we display also the two-dimensional maps $\left(y, p_{t}\right)$ of the asymmetry defined as

$$
A_{ \pm}=\frac{\sigma\left(W^{+}\right)-\sigma\left(W^{-}\right)}{\sigma\left(W^{+}\right)+\sigma\left(W^{-}\right)}
$$

for asymmetric (left panel) and symmetric (right panel) antiquark distributions. The asymmetry so-defined is an asymmetric function under the operation $y \rightarrow-y$ for proton-antiproton collisions and a symmetric one for proton-proton collisions. The STAR collaboration intends to measure the W-boson production in a few year perspective. It is not clear to us whether the map shown in Fig 8 can be studied experimentally in the near future at the Brookhaven RHIC.

\section{Conclusions}

The zeroth order collinear calculation leads to the $\delta$-like distribution in the W-boson transverse momentum. The 1-st order calculation overestimates the experimental data at $p_{t}<5 \mathrm{GeV}$ and underestimates the data at $p_{t}>5 \mathrm{GeV}$. In contrast, already the leading order CCFM approach leads to a reasonable transverse momentum distributions of $\mathrm{W}$-bosons. The small transverse momenta $p_{t}<10 \mathrm{GeV}$ are sensitive to the nonperturbative physics embodied in our calculation in the extra b-dependent form factors. The parameter(s) of the form factor can be adjusted to the experimental low- $p_{t}$ distributions provided the data are sufficiently precise. The simple leading order calculation leaves some room for next-to-leading order contributions at $p_{t}>5-10 \mathrm{GeV}$.

In the present study we have used very simple functional forms of the nonperturbative form factors responsible for intrinsic momentum distribution of partons in colliding hadrons. It is not obvious a priori if these forms are sufficient to describe the variety of data including Drell-Yan dilepton 
production, prompt photon production, heavy quark pair production, etc. This will be a subject of the forthcoming research.

We have studied the dependence of the cross section ratio $W^{-} / W^{+}$in proton-proton collisions on transverse momentum and rapidity of the $\mathrm{W}$ boson. We find a potential to study the $\bar{d}-\bar{u}$ asymmetry at BNL RHIC quantitatively.

There are many other possible ways to study the unintegrated parton distributions further. For instance at RHIC energy the topics to study can be jet or/and particle production [23].

Acknowledgments This paper was completed after Jan Kwieciński passed away. One of us (A.S.) is indebted to Krzysztof Golec-Biernat for a discussion.

\section{References}

[1] B. Abbott et al.(D0 collaboration), Phys. Rev. D61 (2000) 032004.

[2] V.M. Abazov et al. (D0 collaboration), Phys. Lett. B513 (2001) 292.

[3] V.M. Abazov et al. (D0 collaboration), Phys. Lett. B517 (2001) 299.

[4] J.C. Collins, D. Soper and G. Sterman, Nucl. Phys. B250 (1985) 199.

[5] P.B. Arnold and R.P. Kauffman, Nucl. Phys. B349 (1990) 381.

[6] G.A. Ladinsky and C.-P. Yuan, Phys. Rev. D50 (1994) R4239.

[7] R.K. Ellis, D.A. Ross and S. Veseli, Nucl. Phys. B503 (1997) 309.

[8] R.K. Ellis, S. Veseli, Nucl. Phys. B511 (1998) 649.

[9] A. Kulesza, G. Sterman and W. Vogelsang, Phys. Rev. D66 (2002) 014011.

[10] M. Ciafaloni, Nucl. Phys. B296 (1988) 49;

S. Catani, F. Fiorani and G. Marchesini, Phys.Lett.B234 (1990) 339;

Nucl. Phys. B336 (1990) 18.

[11] J. Kwieciński, Acta Phys. Polon. B33 (2002) 1809.

[12] A. Gawron and J. Kwieciński, Acta Phys. Polon. B34 (2003) 133. 
[13] A. Gawron, J. Kwieciński and W. Broniowski, Phys. Rev. D68 (2003) 054001 .

[14] A. Gawron and J. Kwieciński, hep-ph/0309303.

[15] H. Jung and G. Salam, Eur. Phys. J. C19 (2001) 351.

[16] B. Andersson et al. (the Small-x Collaboration), Eur.Phys.J. C25 (2002) 77.

[17] S.P. Baranov, H. Jung, L. Jönsson, S. Padhi and N.P. Zotov, Eur. Phys. J. C24 (2002) 425.

[18] H. Jung, Phys. Rev. D65 (2002) 034015.

[19] H. Jung, Acta Phys.Polon. B33 (2002) 2995;

M. Hansson and H. Jung, hep-ph/0309009.

[20] M. Glück, E. Reya and A. Vogt, Z. Phys. C67 (1995) 433.

[21] M. Glück, E. Reya and A. Vogt, Eur. Phys.J. C5 (1998) 461.

[22] A. Szczurek, V. Uleshchenko, H. Holtmann and J. Speth, Nucl. Phys. A624 (1997) 495.

[23] A. Szczurek, Acta Phys. Polon. B34 (2003) 3191. 


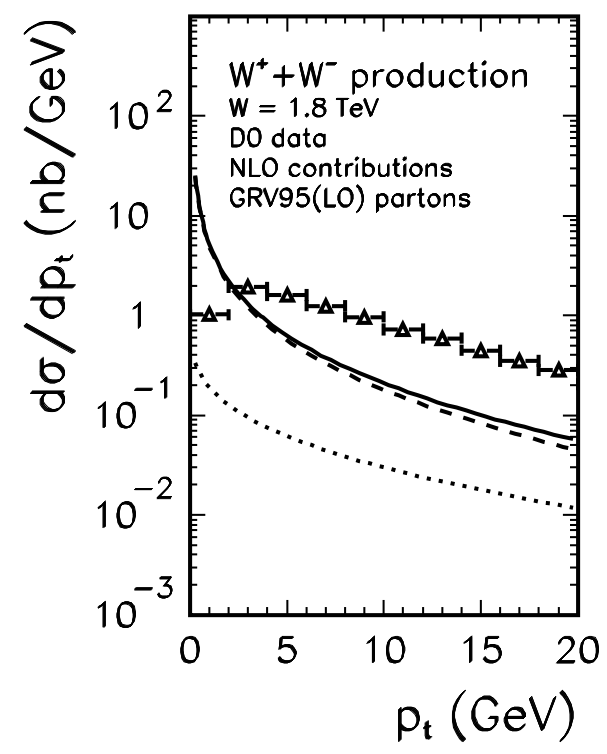

(a)

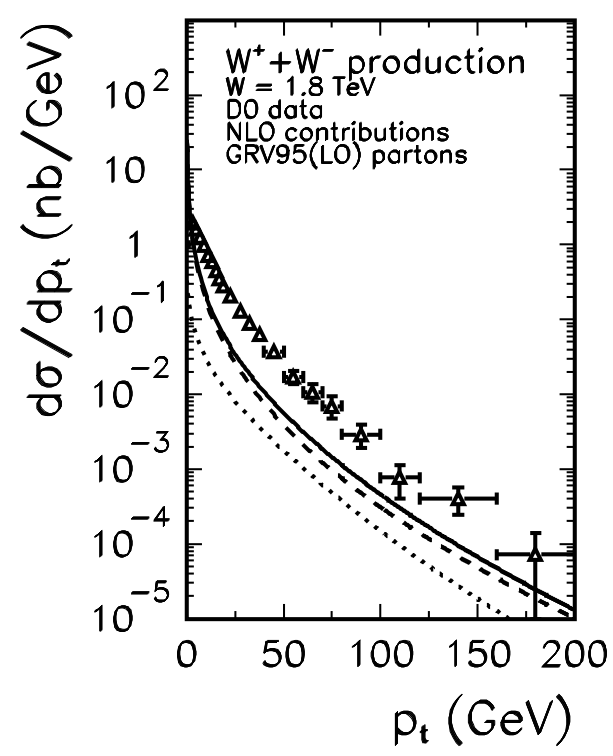

(b)

Figure 1: Transverse momentum distribution of $W^{+}+W^{-}$at $W=1.8 \mathrm{TeV}$. The curves represent the full first-order result (solid) and the annihilation (dashed) and Compton (dotted) components separately. The experimental data are taken from Ref.[3]. 


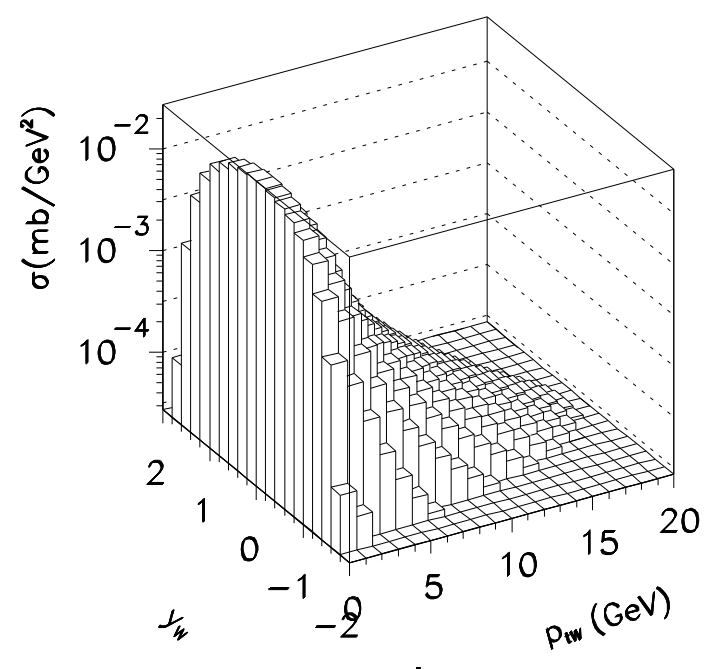

Figure 2: Two-dimensional map of the cross section for $W^{+}+W^{-}$production in proton-antiproton collisions at $W=1.8 \mathrm{TeV}$. In this calculation $b_{0}=1$ $\mathrm{GeV}^{-1}$ (Gaussian form factor). 


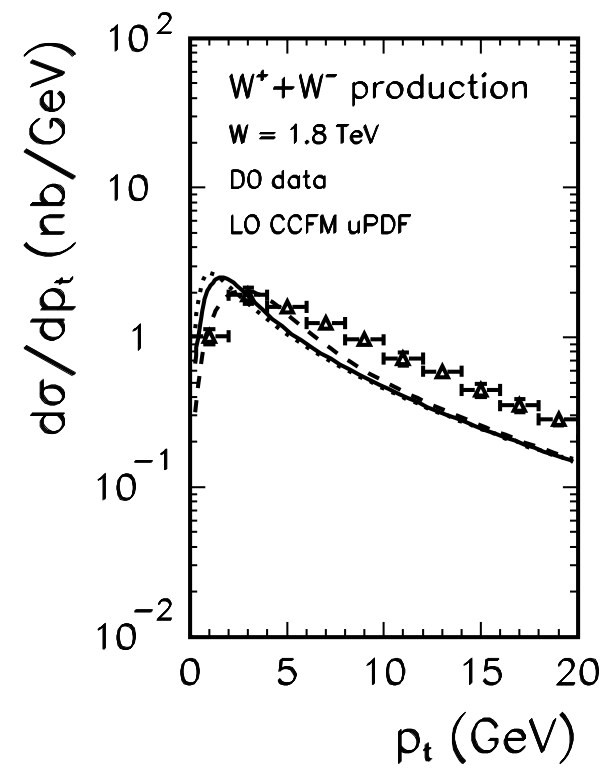

(a)

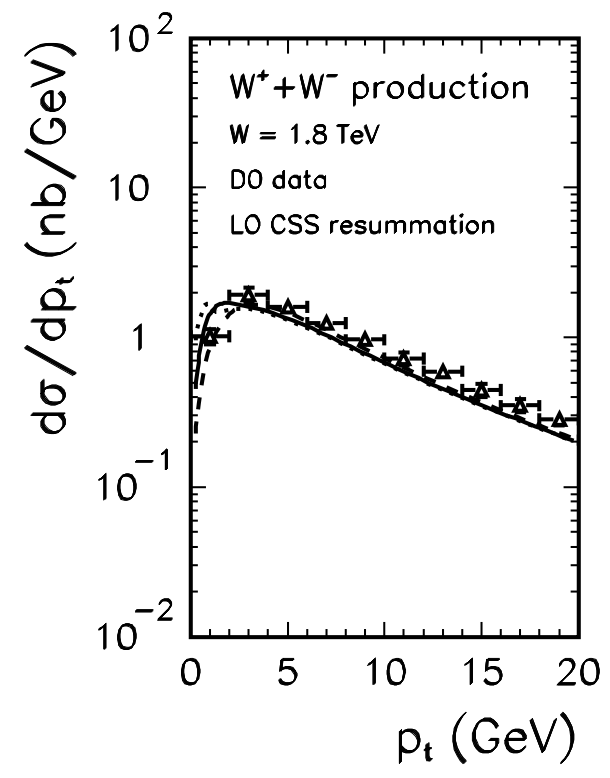

(b)

Figure 3: Transverse momentum distribution of $W^{+}+W^{-}$at $W=1.8 \mathrm{TeV}$ within the formalism of the CCFM uPDF's (left panel) and soft-gluon resummation (right panel) with Gaussian nonperturbative form factor. The three curves correspond to different values of the form factor parameter $b_{0}$ as explained in the text. The experimental data are taken from Ref.[3]. 


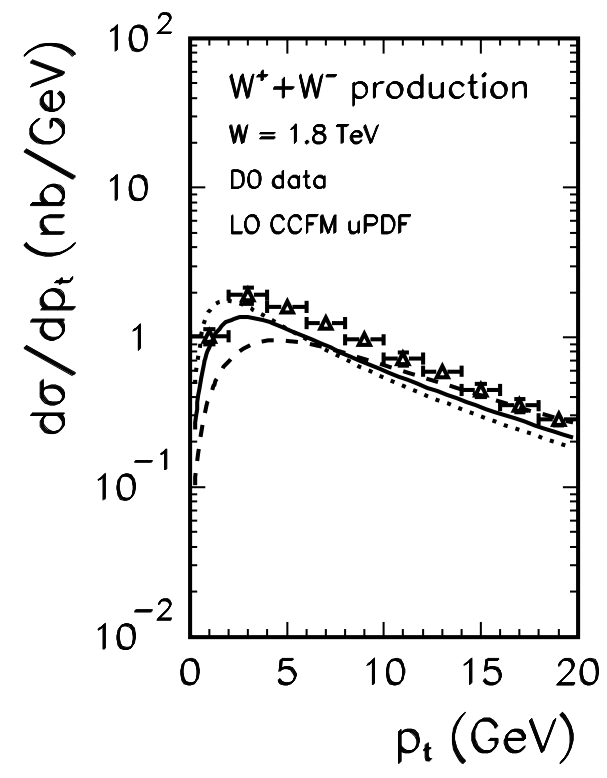

(a)

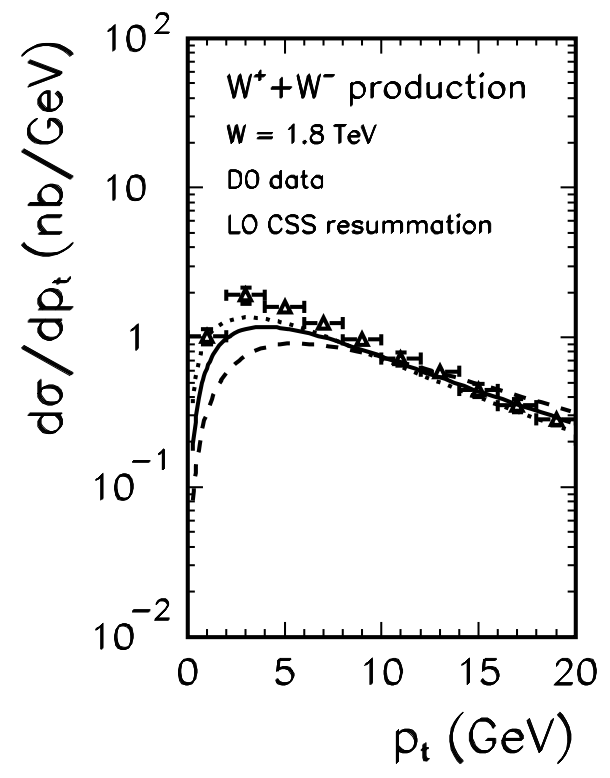

(b)

Figure 4: Transverse momentum distribution of $W^{+}+W^{-}$at $W=1.8 \mathrm{TeV}$ within the formalism of the CCFM uPDF's (left panel) and soft-gluon resummation (right panel) with exponential nonperturbative form factor. The three curves correspond to different values of the form factor parameter $b_{e}$ as explained in the text. The experimental data are taken from Ref.[3]. 


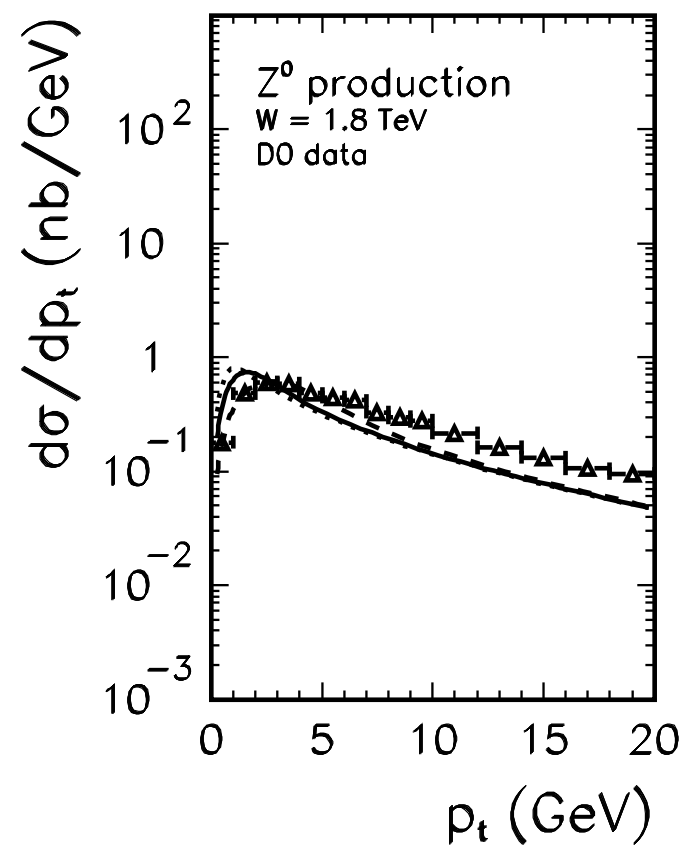

Figure 5: Transverse momentum distribution of $Z^{0}$ at $W=1.8 \mathrm{TeV}$. The three curves correspond to different values of the Gaussian form factor parameter $b_{0}$ as explained in the text. The experimental data are taken from Ref.[1]. 


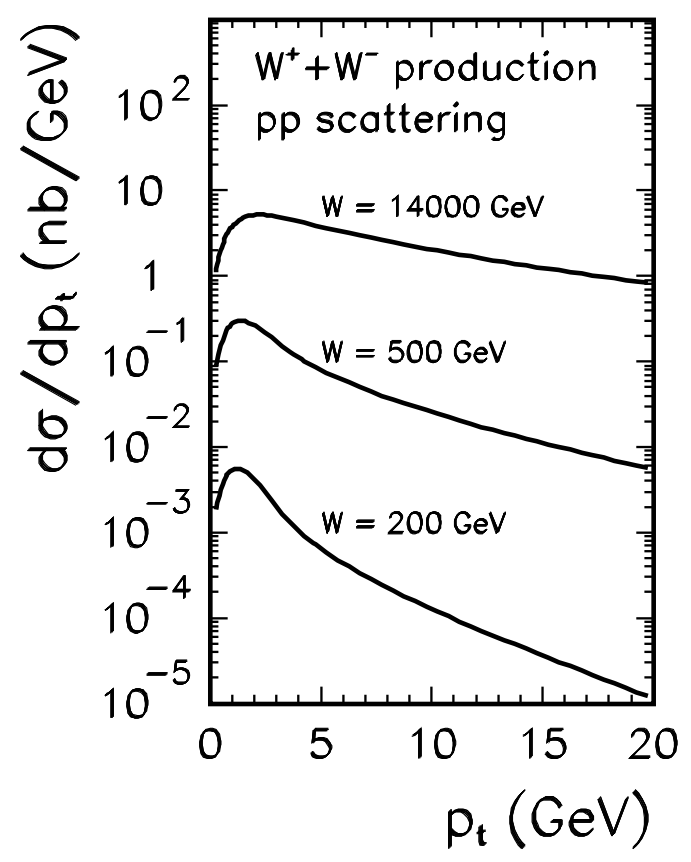

Figure 6: Transverse momentum distribution of $W^{ \pm}$in proton-proton collisions for three different energies. In this calculation $-2<y<2$ and the parameter of the Gaussian form factor $b_{0}=1 \mathrm{GeV}^{-1}$. 


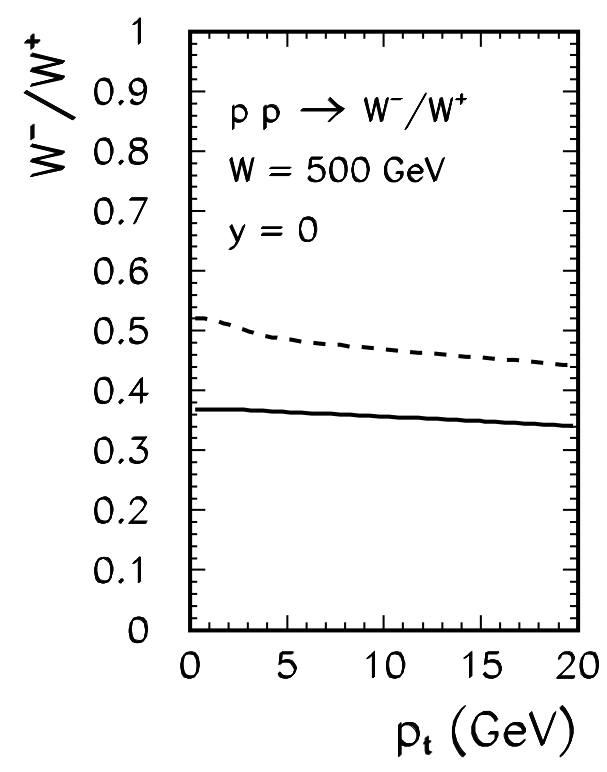

(a)

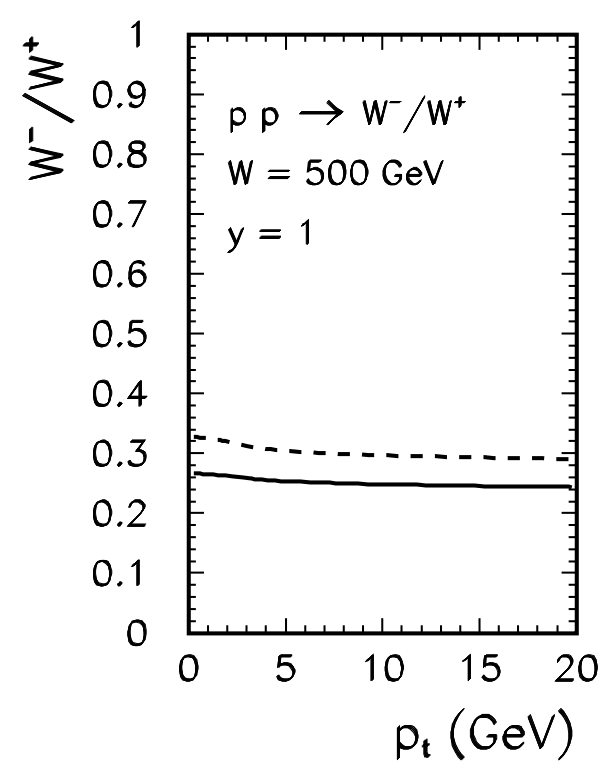

(b)

Figure 7: The ratio $\sigma\left(W^{-}\right) / \sigma\left(W^{+}\right)$for proton-proton collisions at $R H I C$ energy $W=500$ GeV for $y=0$ (left panel) and $y= \pm 1$ (right panel). In this calculation the parameter $b_{0}$ of the nonperturbative Gaussian form factor was fixed for $b_{0}=1 \mathrm{GeV}^{-1}$. The solid line corresponds to calculation with $\bar{d}-\bar{u}$ asymmetry while the dashed line to calculation with symmetric sea. 


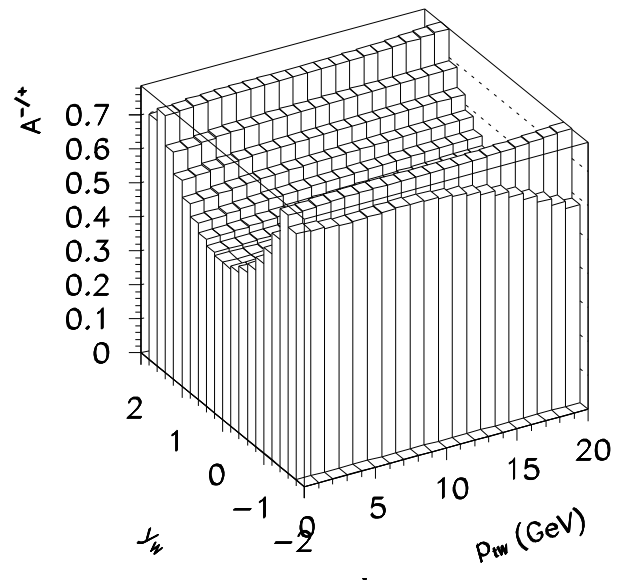

(a)

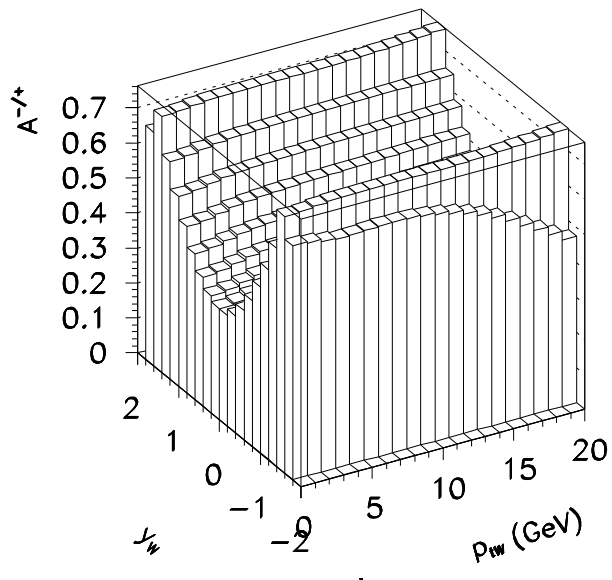

(b)

Figure 8: Two-dimensional maps of the asymmetry $A_{ \pm}$for proton-proton collisions at RHIC energies $W=500 \mathrm{GeV}$ for two cases: in panel (a) with $\bar{d}-\bar{u}$ asymmetry and in panel (b) without the asymmetry. In this calculation the parameter $b_{0}$ of the nonperturbative Gaussian form factor was fixed at $b_{0}$ $=1 \mathrm{GeV}^{-1}$. 